\title{
Relationship between Cognitive Performance and Motor Dysfunction in Patients with Parkinson's Disease: A Pilot Cross-Sectional Study
}

\author{
Valentina Varalta, ${ }^{1}$ Alessandro Picelli, ${ }^{1}$ Cristina Fonte, ${ }^{1}$ Stefania Amato, ${ }^{1}$ Camilla Melotti, \\ Vanja Zatezalo, ${ }^{1}$ Leopold Saltuari, ${ }^{2,3}$ and Nicola Smania ${ }^{1,4}$ \\ ${ }^{1}$ Neuromotor and Cognitive Rehabilitation Research Center, Department of Neurological and Movement Sciences, \\ University of Verona, 37134 Verona, Italy \\ ${ }^{2}$ Department of Neurology, Hochzirl Hospital, 6170 Zirl, Austria \\ ${ }^{3}$ Research Unit for Neurorehabilitation South Tyrol, 39100 Bolzano, Italy \\ ${ }^{4}$ Neurorehabilitation Unit, Hospital Trust of Verona, 37126 Verona, Italy \\ Correspondence should be addressed to Nicola Smania; nicola.smania@univr.it
}

Received 10 October 2014; Revised 19 January 2015; Accepted 18 March 2015

Academic Editor: Matteo Paci

Copyright (c) 2015 Valentina Varalta et al. This is an open access article distributed under the Creative Commons Attribution License, which permits unrestricted use, distribution, and reproduction in any medium, provided the original work is properly cited.

\begin{abstract}
The aim of this pilot cross-sectional study was to extensively investigate the relationships between cognitive performance and motor dysfunction involving balance and gait ability in patients with Parkinson's disease. Twenty subjects with Parkinson's disease underwent a cognitive (outcomes: Frontal Assessment Battery-Italian version, Montreal overall Cognitive Assessment, Trail Making Test, Semantic Verbal Fluency Test, and Memory with Interference Test) and motor (outcomes: Berg Balance Scale, 10-Meter Walking Test, 6-Minute Walking Test, Timed Up and Go Test performed also under dual task condition, and Unified Parkinson's Disease Rating Scale) assessment. Our correlation analyses showed that balance skills are significantly correlated with executive functions, cognitive impairment, and ability to switch attention between two tasks. Furthermore, functional mobility showed a significant correlation with cognitive impairment, verbal fluency, and ability to switch attention between two tasks. In addition, the functional mobility evaluated under the dual task condition showed a significant correlation with cognitive impairment and ability to switch attention between two tasks. These findings might help early identification of cognitive deficits or motor dysfunctions in patients with Parkinson's disease who may benefit from rehabilitative strategies. Future prospective larger-scale studies are needed to strengthen our results.
\end{abstract}

\section{Introduction}

Parkinson's disease (PD) is an idiopathic neurodegenerative disorder caused by a progressive loss of dopaminergic neurons in the substantia nigra pars compacta [1]. Clinical manifestations include reduced amplitude of movement, hypokinesia, rigidity, resting tremor, and postural instability $[1,2]$. The resulting abnormal gait patterns raise the risk of falls, with up to $63 \%$ of people with PD reporting more than one fall per year [3]. In addition to motor symptoms, about $25 \%$ of newly diagnosed PD sufferers present with cognitive deficits [4] frequently involving attention, memory, visuospatial, and executive functions in nondemented people with PD [4-7]. Often, PD-associated cognitive deficits are underestimated in daily clinical practice probably because cognitive assessment is mainly based on the Mini Mental State Examination (MMSE) that has low sensitivity for detecting alterations in specific cognitive and executive functions [8-11].

Internal generation of movement and executive functions both require decision-making processes in order to select an action among several alternative possibilities for the task at hand. The basal ganglia (mostly modulated by dopaminergic projections) seem to have an important role in the mediation 
of cognitive and motor modules to generate an appropriate decision on a resulting action for the task being performed [12]. Patients with PD showed a significant reduction of the dopaminergic projections to the striatum [13]. Previous studies indicate that executive deficits in PD patients without dementia are associated with dysfunction of the caudate nucleus [14-16], suggesting that dopamine is involved in the transfer of information first processed in cognitive brain networks, toward motor-related networks, sequentially [12]. Other potential factors that may influence the relationship between cognitive and motor function in people with $\mathrm{PD}$ are depression and age $[17,18]$.

Although cognitive-motor relationships have been widely described in patients with PD, evaluation takes into consideration a narrow range of outcome measures which do not allow for in-depth complete assessment of cognitive deficits and motor dysfunctions in PD [3, 19-23]. Hence, a closer examination of cognitive-motor relationships is needed to better define to what extent motor aspects depend on cognitive ones and to inform the design of new treatment protocols. Therefore, the aim of this study was to extensively investigate the relationships between cognitive performance and motor dysfunction involving balance and gait ability in patients with PD.

\section{Materials and Methods}

This was a pilot cross-sectional study. Inclusion criteria were confirmed diagnosis of idiopathic PD according to the UK Brain Bank Criteria [24] and MMSE score > 24 [25]. Exclusion criteria were severe dyskinesias or on-off fluctuations, severe comprehension deficit, psychotic disorders, history of alcohol or drug abuse, deficits of somatic sensation involving the lower limbs as assessed by neurological examination, vestibular disorders or paroxysmal vertigo, and other neurological or orthopedic conditions involving the lower limbs such as musculoskeletal diseases, severe osteoarthritis, peripheral neuropathy, and joint replacement. All subjects were outpatients and gave their informed written consent to participate. The study was carried out according to the Declaration of Helsinki and approved by the Local Ethics Committee.

2.1. Evaluation Procedures. During the study period, subjects were instructed to take their PD medications regularly. Each subject underwent the following cognitive and motor assessments during the "on" phase ( 1 to 2.5 hours after having taken their morning dose). The same raters evaluated all subjects (CF and SA performed the cognitive assessment; AP and $\mathrm{VZ}$ performed the motor assessment) in an outpatients clinical setting.

2.1.1. Cognitive Assessment. The main cognitive outcomes were the Frontal Assessment Battery-Italian version (FAB-it) [26] and the Montreal overall Cognitive Assessment (MoCA) [11].

The FAB-it assesses executive functions such as conceptualization, mental flexibility, programming, sensitivity interference, inhibitory control, and environmental autonomy. It consists of 6 tests (similarities, lexical fluency, motor series "Luria" test, conflicting instructions, go/no-go task, and prehension behavior) each rated on a scale from 0 to 3 points. The total score is the sum of all items; the range is from 0 (worst performance) to 18 (best performance) [26].

The MoCA investigates patient's skills in 5 domains: visuospatial/executive, naming, memory, attention, abstraction, and orientation. The total score is the sum of all items, with a maximum score of 30 (best performance) [11].

Other cognitive outcomes were the Trail Making Test (TMT), the Semantic Verbal Fluency Test (SVF), and the Memory with Interference (MI) Test.

Attention capacity was evaluated with the TMT (parts A and B) to assess selective attention, psychomotor speed, and sequencing skills. Part B also investigates the ability to switch attention between two rules or tasks. The time taken to complete the trails is recorded (longer $=$ worse performance) [27].

The SVF assesses verbal fluency by determining the number of words pertaining to a specific semantic category that subjects can spontaneously generate in 2 minutes (higher = better performance) [28].

Working memory was assessed with the MI Test. Subjects are asked to recall a consonant trigram after an interval delay during which they have to count forward starting from a 3-digit number randomly presented by the examiner immediately after the trigram. At the end of an interval delay of 10 seconds, subjects have to recall the trigram. The maximum score is 9 (best performance) [29].

Besides cognitive skills, the patients' mood was evaluated through the Beck Depression Inventory. This tool consists of 21 items rated on a four-point scale of severity focusing on psychological aspects of depression. The total score is the sum of all items; the maximum score is 63 (worst mood) [30].

2.1.2. Motor Assessment. The main motor outcome was performance on the Berg Balance Scale (BBS). This 14-item scale $(0-4$ points/task; best score $=56)$ evaluates balance abilities during sitting, standing, and positional changes [31].

Other motor outcomes were the 10-Meter Walking Test (10MWT), the 6-Minute Walking Test (6MWT), the Timed Up and Go Test (TUG), and the Unified Parkinson's Disease Rating Scale (UPDRS).

The 10MWT was selected as a measure of gait speed $[32,33]$. The subjects were asked to walk on a flat hard floor at their fastest speed for $10 \mathrm{~m}$ without assistance or the use of walking aids (a $10 \mathrm{~m}$ walkway was marked by two lines on the floor at $2 \mathrm{~m}$ and at $8 \mathrm{~m}$ ). In order to minimize acceleration and deceleration, gait speed was measured in the $6 \mathrm{~m}$ between the two marks (timing started when the toes of the leading foot crossed the $2 \mathrm{~m}$ mark and stopped when the toes of the leading foot crossed the $8 \mathrm{~m}$ mark) $[32,33]$. Time was measured using a handheld stopwatch.

Walking capacity was assessed using the 6MWT [34]. The subjects were asked to cover as much ground as possible in 6 min (walking continuously at their possibly fastest speed 
without the use of walking aids) along a marked distance (1 lap, $40 \mathrm{~m}$ ). The distance covered was recorded.

The TUG is functional mobility test associated with balance problems and falls in older adults in which a subject must stand up, walk 3 meters, turn around, walk back, and sit down. The time taken to complete the test is correlated with the level of functional mobility [35]. The subjects performed the TUG under a dual task condition in which they were also asked to count backwards from a randomly selected number between 20 and 100 (TUG-COG) [36].

The UPDRS is a validated tool to follow the longitudinal course of PD. It has 4 subsections. Part III (motor examination) was used (score ranges from 0 to 108 ; high $=$ worst performance) [37].

2.2. Statistical Analysis. Statistical analysis was carried out using the Statistical Package for Social Sciences software, version 20.0, for Macintosh (SPSS Inc, Chicago, IL). Spearman's rank correlation analysis was performed to determine the correlation between motor and cognitive outcomes. Forward stepwise multiple linear regression analyses were performed to further clarify the relationship between cognitive outcomes (the FAB-it, the MoCA, the SVF, and the TMT-B were defined as independent variables), potential confounders (age and the BDI were defined as independent variables), and motor outcomes (the BBS, the TUG, and the TUG-COG were defined as dependent variables). The alpha level for significance was set at $P<0.05$.

\section{Results}

Twenty subjects (12 males, 8 females; mean age $70.3 \pm 6.34$ years; mean years of schooling $10.65 \pm 5.19$ ) presenting with idiopathic PD (mean disease duration $9.88 \pm 5.79$ years) were recruited from among 46 outpatients consecutively admitted to the Neurological Rehabilitation Unit of the Azienda Ospedaliera Universitaria of Verona, Italy. The enrollment period was from January to June 2014. Raw data of patients' performance in all outcome measures are detailed in Table 1.

As to the motor-cognitive correlation in PD, the spearman's analysis showed that the BBS was significantly directly related to the FAB-it $(P<0.001$ and $\rho=0.790)$ and the MoCA $(P=0.015$ and $\rho=0.534)$ and it was significantly inversely related to the TMT-B $(P=0.005$ and $\rho=-0.597)$. The TUG was significantly inversely related to the MoCA $(P=0.045$ and $\rho=-0.564)$ and the SVF $(P<0.006$ and $\rho=-0.713)$ and it was significantly directly related to the TMT-B $(P=0.021$ and $\rho=-0.630)$. The TUG-COG was significantly inversely related to the MoCA $(P=0.025$ and $\rho=-0.667$ ) and significantly directly related to the TMT-B $(P<0.020$ and $\rho=0.683$ ) (see Table 2$)$.

As reported in Table 3, the multiple linear regression analysis showed a significant direct association between the BBS and the FAB-it $(P=0.042 ; \beta=0.640)$.

\section{Discussion}

The aim of this pilot cross-sectional study was to perform an in-depth investigation of the relationship between cognitive
TABLE 1: Raw data of patients' performance in all outcomes.

\begin{tabular}{|c|c|}
\hline $\begin{array}{l}\text { FAB-it ( } 0-18 \text { points) } \\
\text { Median (IQR) }\end{array}$ & $14.00(11.75 ; 16.25)$ \\
\hline $\begin{array}{l}\text { MoCA (0-30 points) } \\
\text { Median (IQR) }\end{array}$ & $22.00(17.75 ; 25.25)$ \\
\hline $\begin{array}{l}\text { TMT-A (seconds) } \\
\text { Mean (SD) }\end{array}$ & $155.20(122.55)$ \\
\hline $\begin{array}{l}\text { TMT-B (seconds) } \\
\text { Mean (SD) }\end{array}$ & 215.47 (103.07) \\
\hline $\begin{array}{l}\text { SVF (number of words) } \\
\text { Median (IQR) }\end{array}$ & $17.50(15.25 ; 22.00)$ \\
\hline $\begin{array}{l}\text { MI (0-9 points) } \\
\text { Median (IQR) }\end{array}$ & $6.00(3.00 ; 7.25)$ \\
\hline $\begin{array}{l}\text { BDI ( } 0-63 \text { points) } \\
\text { Median (IQR) }\end{array}$ & $13.00(7.50 ; 18.00)$ \\
\hline $\begin{array}{l}\text { MMSE ( } 0-30 \text { points) } \\
\text { Median (IQR) }\end{array}$ & $29.00(26.75 ; 30.00)$ \\
\hline $\begin{array}{l}\text { BBS ( } 0-56 \text { points) } \\
\text { Median (IQR) }\end{array}$ & $44.50(38.50 ; 49.00)$ \\
\hline $\begin{array}{l}\text { 10MWT (seconds) } \\
\text { Mean (SD) }\end{array}$ & $9.75(4.65)$ \\
\hline $\begin{array}{l}\text { 6MWT (meters) } \\
\text { Mean (SD) }\end{array}$ & $298.77(96.80)$ \\
\hline $\begin{array}{l}\text { TUG (seconds) } \\
\text { Mean (SD) }\end{array}$ & $11.40(4.84)$ \\
\hline $\begin{array}{l}\text { TUG-COG (seconds) } \\
\text { Mean (SD) }\end{array}$ & $11.96(2.99)$ \\
\hline $\begin{array}{l}\text { UPDRS III (0-108 points) } \\
\text { Median (IQR) }\end{array}$ & $20.50(16 ; 25.50)$ \\
\hline \multicolumn{2}{|c|}{$\begin{array}{l}\text { SD, Standard Deviation; IQR, Interquartile Range; FAB-it, Frontal Assess } \\
\text { ment Battery-Italian version; MoCA, Montreal overall Cognitive Assess } \\
\text { ment; TMT, Trail Making Test; SVF: Semantic Verbal Fluency Test; MI } \\
\text { Memory with Interference; BDI, Beck Depression Inventory; MMSE, Min } \\
\text { Mental State Examination; BBS, Berg Balance Scale; 10MWT, 10-Mete } \\
\text { Walking Test; 6MWT, 6-Minute Walking Test; TUG, Timed Up and Go } \\
\text { TUG-COG, Timed Up and Go under dual task condition; UPDRS III } \\
\text { Unified Parkinson's Disease Rating Scale, part III (motor examination). }\end{array}$} \\
\hline
\end{tabular}

deficits and motor dysfunctions involving balance and gait ability in patients with PD. We found that balance skills (as measured by the BBS) are significantly correlated with executive functions (as measured by the FAB-it), cognitive impairment (as measured by the MoCA), and ability to switch attention between two tasks (as measured by the TMT-B). Furthermore, functional mobility (as measured by the TUG) showed a significant correlation with cognitive impairment (as measured by the MoCA), verbal fluency (as measured by the SVF), and ability to switch attention between two tasks (as measured by the TMT-B). In addition, the functional mobility evaluated under the dual task condition (as measured by the TUG-COG) showed a significant correlation with cognitive impairment (as measured by the MoCA) and ability to switch attention between two tasks (as measured by the TMT-B).

Despite the fact that cognitive-motor relationships have been previously reported in $\mathrm{PD}[3,19-23]$, many of these studies did not extensively investigate motor or cognitive functions [3, 19, 22, 23]. For example, Lee and colleagues 
TABLE 2: Correlation matrix for study variables (Spearman's rho).

\begin{tabular}{|c|c|c|c|c|c|c|c|c|c|c|c|c|c|}
\hline $\begin{array}{l}\text { Outcome } \\
\text { measures }\end{array}$ & FAB-it & $\mathrm{MoCA}$ & TMT-A & TMT-B & SVF & MI & BDI & BBS & $10 \mathrm{MWT}$ & $6 \mathrm{MWT}$ & TUG & TUG-COG & UPDRS III \\
\hline FAB-it & 1.000 & & & & & & & & & & & & \\
\hline $\mathrm{MoCA}$ & $0.715^{*}$ & 1.000 & & & & & & & & & & & \\
\hline TMT-A & $-0.517^{*}$ & $-0.776^{*}$ & 1.000 & & & & & & & & & & \\
\hline TMT-B & $-0.704^{*}$ & $0.822^{*}$ & $0.740^{*}$ & 1.000 & & & & & & & & & \\
\hline SVF & $0.583^{*}$ & $0.778^{*}$ & $-0.579^{*}$ & $-0.729^{*}$ & 1.000 & & & & & & & & \\
\hline MI & $0.596^{*}$ & $0.664^{*}$ & $-0.511^{*}$ & $-0.553^{*}$ & $0.736^{*}$ & 1.000 & & & & & & & \\
\hline BDI & 0.001 & -0.106 & 0.223 & 0.030 & 0.133 & -0.029 & 1.000 & & & & & & \\
\hline BBS & $0.790^{*}$ & $0.534^{*}$ & -0.304 & $-0.597^{*}$ & 0.371 & 0.283 & -0.114 & 1.000 & & & & & \\
\hline 10MWT & -0.138 & -0.090 & 0.114 & 0.308 & -0.313 & -0.146 & -0.371 & -0.169 & 1.000 & & & & \\
\hline $6 \mathrm{MWT}$ & 0.149 & 0.058 & -0.129 & -0.095 & 0.292 & 0.082 & -0.014 & 0.429 & -0.715 & 1.000 & & & \\
\hline TUG & -0.372 & $-0.564^{*}$ & 0.477 & $0.630^{*}$ & $-0.713^{*}$ & -0.243 & -0.102 & -0.499 & $0.762^{*}$ & $-0.709^{*}$ & 1.000 & & \\
\hline TUG-COG & 0.190 & $-0.667^{*}$ & 0.392 & $0.683^{*}$ & -0.538 & 0.101 & -0.192 & -0.303 & 0.228 & 0.027 & $0.727^{*}$ & 1.000 & \\
\hline UPDRS III & -0.281 & -0.035 & -0.072 & 0.077 & 0.088 & 0.272 & -0.103 & -0.427 & -0.029 & 0.135 & 0.074 & $0.606^{*}$ & 1.000 \\
\hline
\end{tabular}

FAB-it, Frontal Assessment Battery-Italian version; MoCA, Montreal overall Cognitive Assessment; TMT, Trail Making Test; SVF, Semantic Verbal Fluency; MI, Memory with Interference Test; BDI, Beck Depression Inventory; BBS, Berg Balance Scale; 10MWT, 10-Meter Walking Test; 6MWT, 6-Minute Walking Test; TUG, Timed Up and Go Test; TUG-COG, Timed Up and Go Test under dual task condition; UPDRS III, Unified Parkinson's Disease Rating Scale part III (motor examination).

${ }^{*}$ Significant correlation $(P<0.05)$.

TABLE 3: Multiple linear regression analysis.

\begin{tabular}{|c|c|c|c|c|c|c|}
\hline \multirow{2}{*}{ Dependent variables } & \multicolumn{6}{|c|}{ Independent variables } \\
\hline & FAB-it & MoCA & TMT-B & SVF & Age & BDI \\
\hline \multicolumn{7}{|l|}{ BBS } \\
\hline$\beta$ & 0.640 & 0.269 & -0.117 & -0.309 & 0.027 & 0.094 \\
\hline$P$ & $0.042^{*}$ & 0.481 & 0.745 & 0.322 & 0.892 & 0.635 \\
\hline \multicolumn{7}{|l|}{ TUG } \\
\hline$\beta$ & -0.661 & -0.289 & -0.086 & 0.126 & -0.124 & -0.084 \\
\hline$P$ & 0.259 & 0.704 & 0.908 & 0.814 & 0.748 & 0.821 \\
\hline \multicolumn{7}{|l|}{ TUG-COG } \\
\hline$\beta$ & 0.148 & -0.719 & -0.229 & -0.548 & -0.322 & 0.005 \\
\hline$P$ & 0.690 & 0.223 & 0.679 & 0.320 & 0.307 & 0.991 \\
\hline
\end{tabular}

FAB-it, Frontal Assessment Battery-Italian version; MoCA, Montreal overall Cognitive Assessment; TMT, Trail Making Test; SVF, Semantic Verbal Fluency; BDI, Beck Depression Inventory; BBS, Berg Balance Scale; TUG, Timed Up and Go Test; TUG-COG, Timed Up and Go Test under dual task condition.

${ }^{*}$ Significant correlation $(P<0.05)$.

examined only the postural instability skill. Specifically they investigated the relationship between postural instability, as measured by computerized dynamic posturography, and cognitive impairment and found a correlation between balance abilities and MMSE scores. They demonstrated a significant correlation between equilibrium scores and visuospatial and memory functions [22]. In an earlier study, Yogev and coworkers investigated only the relationship between gait ability and cognitive function. They found that executive function measures were significantly correlated with gait variability during dual tasking [23]. Allcock and colleagues, regarding cognitive abilities, investigated only attention skills and found an association between fall frequency and attention [3]. Williams and coworkers' study correlated motor abilities only with cognitive screening test scores, finding a relation between postural/gait instability and the MMSE [19].
Unlike these studies, more recent research has investigated other aspects of cognitive and motor performance in patients with PD and analyzed their correlations [20, 21]. Specifically, Domellöf and colleagues explored which aspects of cognition (memory, psychomotor speed, attention, language, visuospatial abilities, and executive functions) are connected to different motor signs as investigated by the UPDRS [20]. They found that bradykinesia was associated with executive functions (working memory and mental flexibility), whereas axial signs (such as postural instability, gait disturbances, and bulbar dysfunctions) were associated with memory and visuospatial abilities [20]. Similarly, Poletti and colleagues reported that the bradykinesia score on the UPDRS predicted performances on the executive tasks. But differently from Domellöf and colleagues' study, correlation analyses revealed that axial signs were also associated 
with executive deficits [21]. This result is in line with our data. Poletti and colleagues evaluated balance performance according to the UPDRS. It should be noted, however, that the UPDRS is a qualitative test that may be inadequate for accurately estimating balance and gait performance due to the subjective nature of the evaluation and the lack of normative criteria $[38,39]$. Therefore, scales that yield a more varied estimate of posture and gait control are needed to obtain a better overall estimate of quantitative postural control, as we have done. Furthermore, this aspect could explain the differences in results between Domellöf and colleagues' and Poletti and coworkers' studies.

Similarly important is to extensively evaluate cognitive skills in patients with PD. In our view, the cognitive performance of patients with PD should be assessed by specific and appropriate cognitive measures in addition to the MMSE, considering that this examination alone does not allow identifying specific deficits in executive functions that are usually impaired in patients with PD [8-10].

Interestingly, our regression analyses showed a significant association between balance skills (as measured by the BBS) and executive functions (as measured by the FAB-it). From a rehabilitation point of view, it may be useful to clarify these aspects of the association between cognitive deficits and motor dysfunction in patients with PD in order to develop more appropriate rehabilitation programs that also include ecological situations for training motor and cognitive functions. In this context, prospective studies are needed to further investigate the effects of cognitive training on motor performance.

This study has several limitations. The main one is the small sample size that may have missed some aspects of the relationship between cognitive impairment and motor disorders in the correlation and regression analyses. Second, we excluded patients with MMSE score < 24; thus our population and results are limited to a relatively normal cognitive function for age. Third, we did not assess memory functions. Fourth, because we did not investigate patients in the "off" phase, we cannot draw conclusions about cognitivemotor relationships in the unmedicated state. Fifth, we did not include instrumental evaluations of gait and balance parameters.

\section{Conclusions}

Our findings have some clinical implications. Indeed, they may help early identification of cognitive deficits or motor dysfunctions in patients with PD who may benefit from rehabilitative strategies. Future prospective larger-scale studies including other instrumental motor outcomes are needed to strengthen our results and better explore the effects of training on cognitive-motor relationships in patients with PD.

\section{Conflict of Interests}

The authors received no financial support for the research or authorship of this paper. No commercial party having a direct financial interest in the results of the research supporting this paper has or will confer a benefit on the authors or on any organization with which the authors are associated.

\section{References}

[1] J. Meara and B. K. Bhowmick, "Parkinson's disease and Parkinsonism in the elderly: introduction," in Parkinson's Disease and Parkinsonism in the Elderly, J. Meara and W. C. Koller, Eds., Cambridge University Press, Cambridge, UK, 2000.

[2] A. Picelli, M. Camin, M. Tinazzi et al., "Three-dimensional motion analysis of the effects of auditory cueing on gait pattern in patients with Parkinson's disease: a preliminary investigation," Neurological Sciences, vol. 31, no. 4, pp. 423-430, 2010.

[3] L. M. Allcock, E. N. Rowan, I. N. Steen, K. Wesnes, R. A. Kenny, and D. J. Burn, "Impaired attention predicts falling in Parkinson's disease," Parkinsonism \& Related Disorders, vol. 15, no. 2, pp. 110-115, 2009.

[4] D. Muslimović, B. Post, J. D. Speelman, and B. Schmand, "Cognitive profile of patients with newly diagnosed Parkinson disease," Neurology, vol. 65, no. 8, pp. 1239-1245, 2005.

[5] E. Elgh, M. Domellöf, J. Linder, M. Edström, H. Stenlund, and L. Forsgren, "Cognitive function in early Parkinson's disease: a population-based study," European Journal of Neurology, vol. 16, no. 12, pp. 1278-1284, 2009.

[6] T. Foltynie, C. E. G. Brayne, T. W. Robbins, and R. A. Barker, "The cognitive ability of an incident cohort of Parkinson's patients in the UK. The CamPaIGN study," Brain, vol. 127, no. 3, pp. 550-560, 2004.

[7] E. Mamikonyan, P. J. Moberg, A. Siderowf et al., "Mild cognitive impairment is common in Parkinson's disease patients with normal Mini-Mental State Examination (MMSE) scores," Parkinsonism \& Related Disorders, vol. 15, no. 3, pp. 226-231, 2009.

[8] Y. Dong, V. K. Sharma, B. P.-L. Chan et al., "The Montreal Cognitive Assessment (MoCA) is superior to the Mini-Mental State Examination (MMSE) for the detection of vascular cognitive impairment after acute stroke," Journal of the Neurological Sciences, vol. 299, no. 1-2, pp. 15-18, 2010.

[9] C. A. Meyers and J. S. Wefel, "The use of the mini-mental state examination to assess cognitive functioning in cancer trials: no ifs, ands, buts, or sensitivity," Journal of Clinical Oncology, vol. 21, no. 19, pp. 3557-3558, 2003.

[10] K. Gershman, D. K. Onion, and D. L. Meyer, "Executive function testing to diagnose, subtype dementias," American Family Physician, vol. 73, no. 2, pp. 211-212, 2006.

[11] Z. S. Nasreddine, N. A. Phillips, V. Bédirian et al., "The montreal cognitive assessment, MoCA: a brief screening tool for mild cognitive impairment," Journal of the American Geriatrics Society, vol. 53, no. 4, pp. 695-699, 2005.

[12] A. Nagano-Saito, K. Martinu, and O. Monchi, "Function of basal ganglia in bridging cognitive and motor modules to perform an action," Frontiers in Neuroscience, vol. 8, no. 8, article 187, 2014.

[13] A. Samii, J. G. Nutt, and B. R. Ransom, "Parkinson's disease," The Lancet, vol. 363, no. 9423, pp. 1783-1793, 2004.

[14] S. J. G. Lewis, A. Dove, T. W. Robbins, R. A. Barker, and A. M. Owen, "Cognitive impairments in early Parkinson's disease are accompanied by reductions in activity in frontostriatal neural circuitry," The Journal of Neuroscience, vol. 23, no. 15, pp. 6351$6356,2003$. 
[15] O. Monchi, M. Petrides, J. Doyon, R. B. Postuma, K. Worsley, and A. Dagher, "Neural bases of set-shifting deficits in Parkinson's disease," The Journal of Neuroscience, vol. 24, no. 3, pp. 702 710, 2004.

[16] O. Monchi, M. Petrides, B. Mejia-Constain, and A. P. Strafella, "Cortical activity in Parkinson's disease during executive processing depends on striatal involvement," Brain, vol. 130, no. 1, pp. 233-244, 2007.

[17] P. S. Foster, R. C. Yung, V. Drago, G. P. Crucian, and K. M. Heilman, "Working memory in Parkinson's disease: the effects of depression and side of onset of motor symptoms," Neuropsychology, vol. 27, no. 3, pp. 303-313, 2013.

[18] J. W. Kim, S. M. Cheon, M. J. Park, S. Y. Kim, and H. Y. Jo, "Cognitive impairment in Parkinson's disease without dementia: subtypes and influences of age," Journal of Clinical Neurology, vol. 5, no. 3, pp. 133-138, 2009.

[19] L. N. Williams, P. Seignourel, G. P. Crucian et al., "Laterality, region, and type of motor dysfunction correlate with cognitive impairment in Parkinson's disease," Movement Disorders, vol. 22, no. 1, pp. 141-145, 2007.

[20] M. E. Domellöf, E. Elgh, and L. Forsgren, “The relation between cognition and motor dysfunction in drug-naive newly diagnosed patients with Parkinson's disease," Movement Disorders, vol. 26, no. 12, pp. 2183-2189, 2011.

[21] M. Poletti, D. Frosini, C. Pagni et al., "Mild cognitive impairment and cognitive-motor relationships in newly diagnosed drug-naive patients with Parkinson's disease," Journal of Neurology, Neurosurgery and Psychiatry, vol. 83, no. 6, pp. 601-606, 2012.

[22] J. M. Lee, S.-B. Koh, S. W. Chae et al., "Postural instability and cognitive dysfunction in early Parkinson's disease," The Canadian Journal of Neurological Sciences, vol. 39, no. 4, pp. 473-482, 2012.

[23] G. Yogev, N. Giladi, C. Peretz, S. Springer, E. S. Simon, and J. M. Hausdorff, "Dual tasking, gait rhythmicity, and Parkinson's disease: which aspects of gait are attention demanding?" European Journal of Neuroscience, vol. 22, no. 5, pp. 1248-1256, 2005.

[24] A. J. Hughes, S. E. Daniel, L. Kilford, and A. J. Lees, "Accuracy of clinical diagnosis of idiopathic Parkinson's disease: a clinicopathological study of 100 cases," Journal of Neurology Neurosurgery \& Psychiatry, vol. 55, no. 3, pp. 181-184, 1992.

[25] M. F. Folstein, S. E. Folstein, and P. R. McHugh, "Mini-mental state: a practical method for grading the cognitive state of patients for the clinician," Journal of Psychiatric Research, vol. 12, no. 3, pp. 189-198, 1975.

[26] I. Appollonio, M. Leone, V. Isella et al., “The frontal assessment battery (FAB): normative values in an Italian population sample," Neurological Sciences, vol. 26, no. 2, pp. 108-116, 2005.

[27] A. R. Giovagnoli, M. Del Pesce, S. Mascheroni, M. Simoncelli, M. Laiacona, and E. Capitani, "Trail Making Test: normative values from 287 normal adult controls," The Italian Journal of Neurological Sciences, vol. 17, no. 4, pp. 305-309, 1996.

[28] H. Spinnler and G. Tognoni, "Standardizzazione e taratura di test neuropsicologici," The Italian Journal of Neurological Sciences, vol. 8, supplement 6, pp. 1-20, 1987.

[29] S. Mondini, D. Mapelli, A. Vestri, G. Arcara, and P. S. Bisiacchi, Esame Neuropsicologico Breve 2, Raffaello Cortina Editore, 2011.

[30] A. T. Beck, C. H. Ward, M. Mendelson, J. Mock, and J. Erbaugh, "An inventory for measuring depression," Archives of General Psychiatry, vol. 4, pp. 561-571, 1961.
[31] K. Berg, S. Wood-Dauphinee, and J. I. Williams, “The balance scale: reliability assessment with elderly residents and patients with an acute stroke," Scandinavian Journal of Rehabilitation Medicine, vol. 27, no. 1, pp. 27-36, 1995.

[32] R. W. Bohannon, A. W. Andrews, and M. W. Thomas, "Walking speed: reference values and correlates for older adults," Journal of Orthopaedic and Sports Physical Therapy, vol. 24, no. 2, pp. 86-90, 1996.

[33] R. W. Bohannon, "Comfortable and maximum walking speed of adults aged 20-79 years: reference values and determinants," Age and Ageing, vol. 26, no. 1, pp. 15-19, 1997.

[34] P. L. Enright, “The six-minute walk test," Respiratory Care, vol. 48, no. 8, pp. 783-785, 2003.

[35] S. Morris, M. E. Morris, and R. Iansek, "Reliability of measurements obtained with the Timed 'Up \& Go' test in people with Parkinson disease," Physical Therapy, vol. 81, no. 2, pp. 810-818, 2001.

[36] A. Shumway-Cook, S. Brauer, and M. Woollacott, "Predicting the probability for falls in community-dwelling older adults using the timed up \& go test," Physical Therapy, vol. 80, no. 9, pp. 896-903, 2000.

[37] J. Song, B. E. Fisher, G. Petzinger, A. Wu, J. Gordon, and G. J. Salem, "The relationships between the unified Parkinson's disease rating scale and lower extremity functional performance in persons with early-stage Parkinson's disease," Neurorehabilitation and Neural Repair, vol. 23, no. 7, pp. 657-661, 2009.

[38] A. L. Adkin, J. S. Frank, and M. S. Jog, "Fear of falling and postural control in Parkinson's disease," Movement Disorders, vol. 18, no. 5, pp. 496-502, 2003.

[39] B. R. Bloem, D. J. Beckley, B. J. van Hilten, and R. A. C. Roos, "Clinimetrics of postural instability in Parkinson's disease," Journal of Neurology, vol. 245, no. 10, pp. 669-673, 1998. 


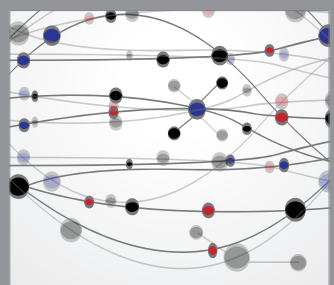

The Scientific World Journal
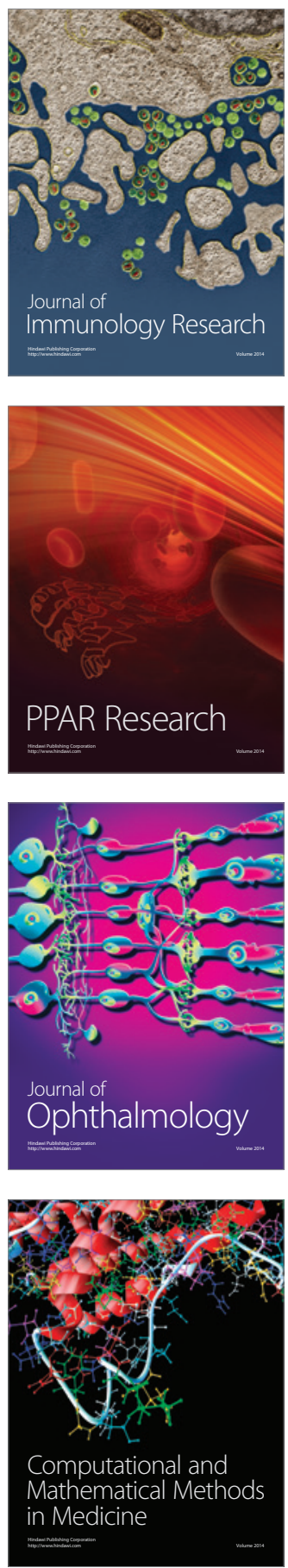

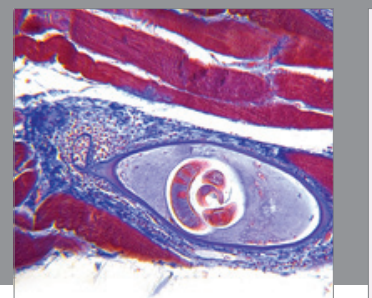

Gastroenterology

Research and Practice
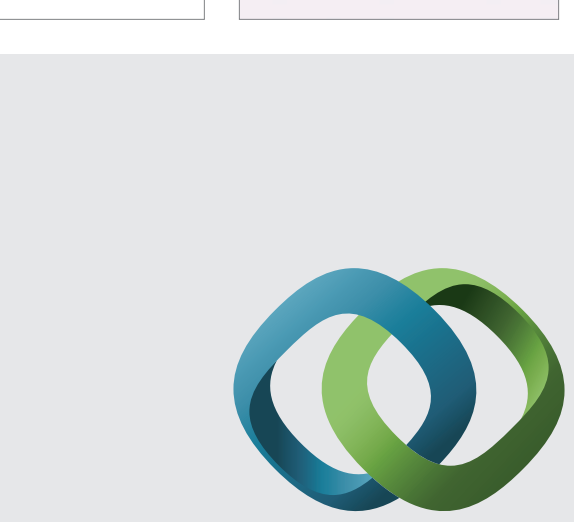

\section{Hindawi}

Submit your manuscripts at

http://www.hindawi.com
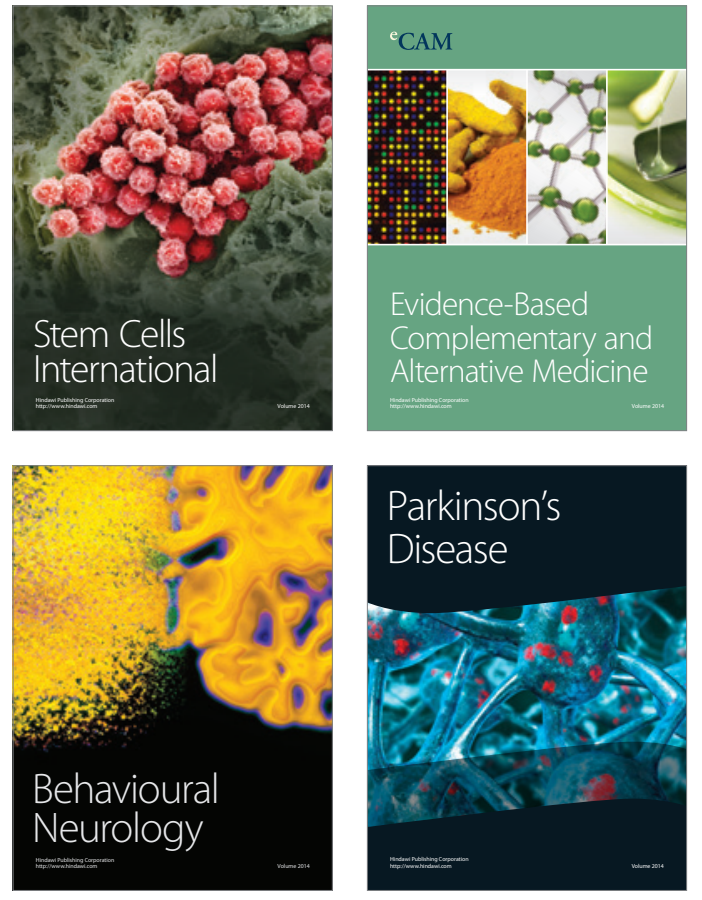
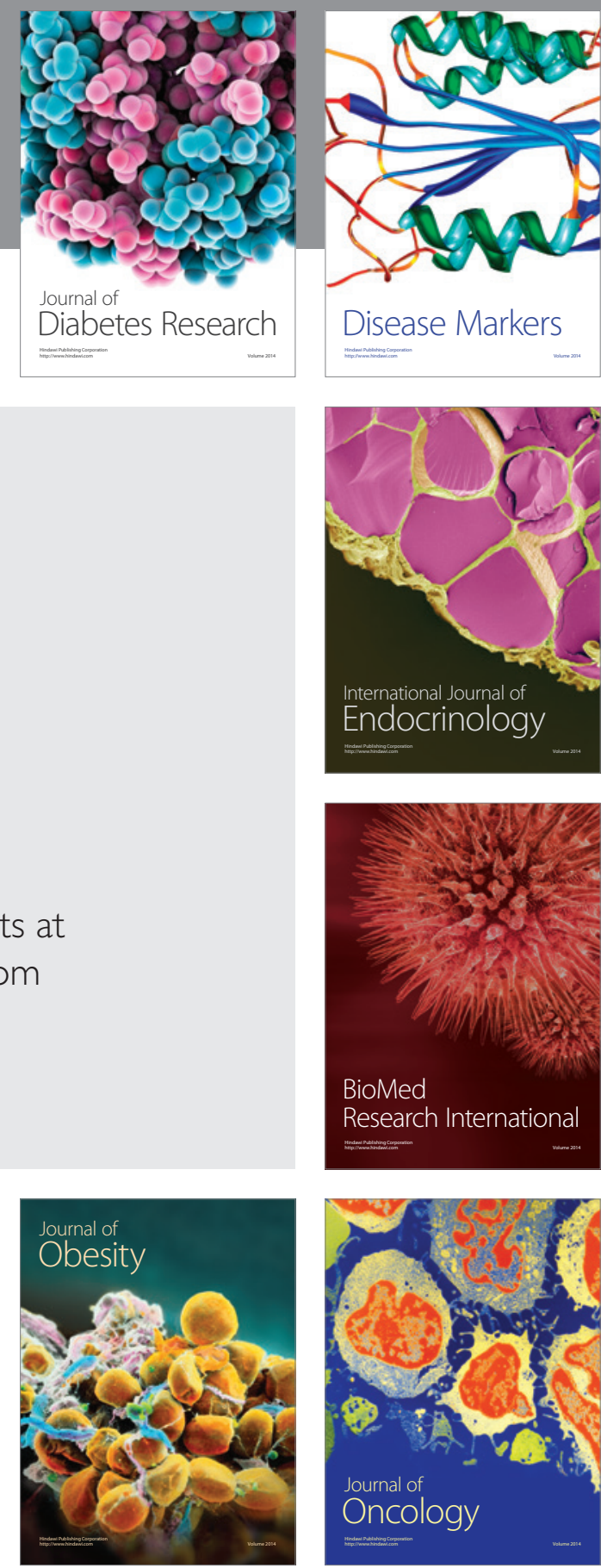

Disease Markers
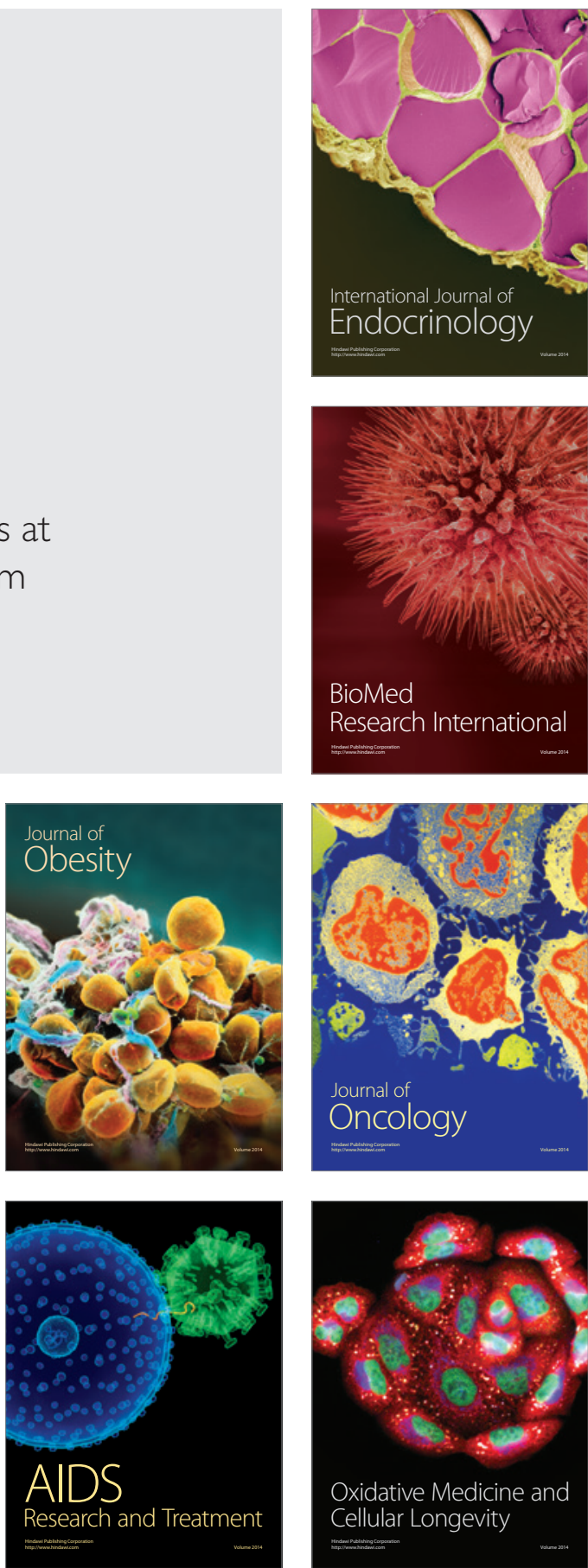